\title{
SMOKING IS ONLY ON A PARTIAL BAN, WHAT ABOUT TOTAL BAN?
}

\section{Short communication}

Tobacco smoking is already labeled the most hazardous risk factor responsible for humans' morbidity and mortality. It comes on top of other high risk diseases like diabetes, hypertension, and dyslipidemia because, it is considered as number one link for the top fatalities from: cerebral-vascular strokes, ischemic heart diseases and acute myocardial infarctions.

Smoking is responsible (up to $90 \%$ ) for cancers cases of the respiratory system that includes cancer lung as, number one cause of cancers' deaths in both males and, females of the US and some countries. This includes too, the higher risk for lips, tongue, laryngeal, and bronchial carcinomas. Recently, some studies had linked smoking even to the cancer breast of females known before as the top fatal one.

Its concentrated forms are the: Clay-pipe smoking (Shishah) which is worldwide popular, especially in countries of "Mediterranean-Sea area", with rising numbers among female users. It is even about hundred times more injurious than a regular cigarette for hazardous effects starting from cancer lips, tongue, larynx and bronchi, in addition, to the effects on the rest of body systems. "AlKat" is another plant with much higher percentage of nicotine than tobacco and is mainly planted for chewing and smoking, mainly in Yemen and nearby countries. Unfortunately, for the sake of habitual misuse, many other forms were also documented cultivated since 'Pharoanic Era' of history till today, by all worldwide continents.

Smoking is also responsible, for morbidity of body systems such as: a- Gastro-intestinal system: inducing or enhancing: gastric ulcers and carcinomas, liver \& pancreatic carcinomas. Crohn's disease is three folds more in smokers, but this ratio is uniquely and paradoxically inversed for ulcerative colitis. b- Genito-urinary system: accused in renal \& bladder carcinomas, cancer cervix of females \& prostatic carcinoma in males. It potentiates renal ischemia \& chronic renal diseases deteriorating progressively glomerular filtration rate, thus aggravating diabetes and hypertension effect especially in an elderly heavy smoker patient taking multiple medications, so, enhancing the renal failure and renal replacement therapy by dialysis or transplantation. c- CNS with excessive habituation, dependency, depression \& anxiety neurosis, with worsening of other co-existing CNS diseases as Parkinsonism, and potential ischemic strokes. d- Vessels of peripheral blood system causing ischemia, and thus can potentiate serious diseases as: poor wound healing, Beurger's disease mainly a disease of the middle-age affecting the peripheral neuro-vascular bundle that can cause potential gangrene of the limbs and possible amputation, if smoking is not totally stopped as also worsen the peripheral vascular occlusive (ischemic) disease effect by presence of other co-existing diseases as diabetes, dyslipidemia, hypertension or a vasculitis. This may end into one of the lengthy major surgical arterial bypass grafts operation which carries significant failure, morbidity, or mortality rates.

Pregnant smokers females are predisposed to abortions, intra-uterine, growth retardation, preterm-birth, congenital heart diseases, especially, if associated with excessive coffee and herbal tea intake, or with alcoholism that adds more to that too. Among all women, more females in the US and some other countries started to actively smoke heavily in rising numbers by the last three decades.

Secondary (passive) smokers by attendants of a nearby smoker still carry potential significant hazards and cancerous effect, too as bronchitis, bronchial asthma, and cancer lung with cardiovascular strokes and myocardial infarctions potentiating effects, too. It is also annoying to others in business areas, closed places or shops and means of transportations as trains, buses, metros.

Smoke of one cigarette contains by analysis four thousand chemical compounds and 
at least about 400 poisonous substances like: 1- Acetone: used mainly as nail polish, 2Ammonia: used mainly as a detergent for floors and bathrooms, 3- Formalin: a preservative for the dead bodies and laboratory sample specimens, 4- Cadmium: used mainly in re-charging batteries, 5- Carbon monoxide: comes out from exhaust of gas furnaces and cars, 6- Butane: used as a liquid in a cigarette lighter, 7- Chloride phenol: used in manufacturing plastic-pipes, 8- DDT (dichloro-diphenyl-trichloroethane) used mainly as an insecticide, 9- Arsenic: a poison, 10Naphthalene: used as naphthalene balls in storing clothes as an insecticide, 11- Nicotine: a habitual-to-addictive substance, 12Methanol: a gas used for rockets, 13- Acetylene: a colorless gas used in welding, 14Aniline: an oil liquid poisonous extracted from coal tar, used in perfumes and dyes, 15- Anthracene: a hydrocarbonic substance in coal tar, 16- Argon: a colorless, odorless element in the air and volcanoes, used specifically to fill electric lamps and electronic gas pipes, 17- Benzene, or Benzil: an inflammable gas manufactured from coal tar and used in manufacture of saccharine, aspirin, 18- Butadiene: an inflammable hydrocarbonic gas used in manufacturing artificial rubber, 19- Methane gas present in stagnant

Major Human Carcinogens an

- Acetaldehyde: about 980 micrograms- 1.37 milligrams,

- 4-Aminobiphenyl: 0.2- 23 nanograms,

- Benzene : 5.9-75 micrograms,

- Cadmium: 1.7 micrograms,

- Hydrazine: 32 micrograms,

- Isoprene: 3.1 milligrams,

- 2-Napthylamine: 1.5-35 nanograms,

Numbers are strikingly high as to smoking endemicity. There are about $20 \%$ of youth population aged 15-30 years who smoke. Numbers are even reaching more than $30 \%$ by youth of under-developed countries and certain areas of the developed countries. Many persons deny associated smoking of other substances like Cannabis (Hash), Marijuana, Cocaine (Cracks), or drug cocktails made of barbiturates, amphetamine derivatives, hallucinogens, or psychotropic medi- water and mines, 20- Stearic acid; a whitish solid substance extracted from some fats and used in candles' manufacturing, 21- Toluene: a colorless liquid similar to benzene extracted from coal tar and others to be used in manufacture of drugs and dyes, 22- Toluedene: an amino-compound extracted from Toluene and used in dyes and drug manufacturing, 23- Acetaldehyde: a colorless liquid vapor perfume, 24- Acetic Acid: Vinegar or, Acid of vinegar, 25-Alfran: A colorless inflammable liquid, 26- Indol: a crystal compound, 27- Isopropane: an inflammable hydro carbonic liquid, 28- Linoleic Acid: A flax seed acid, 29- Methylamine: an explosive flammable gas, 30- Nitric oxide: a poisonous gas, 30- Benzene nitrite: a poisonous gas, 31- Hydrogen cyanide: a poisonous substance, 32-Quinoline: a type of oil or fats, and 33- Sitosterol: a type of complex fats,

The above data should be mentioned to patients during their treatment sessions in a simple scientific way by establishing good rapport with them, to get them aversively deviated from smoking. The magnified hazardous effects of both the clay-pipe (Shisha) and (Al-Kat); should also be explained adequately to all concerned patients and their active co-smoking friends $\&$ families.

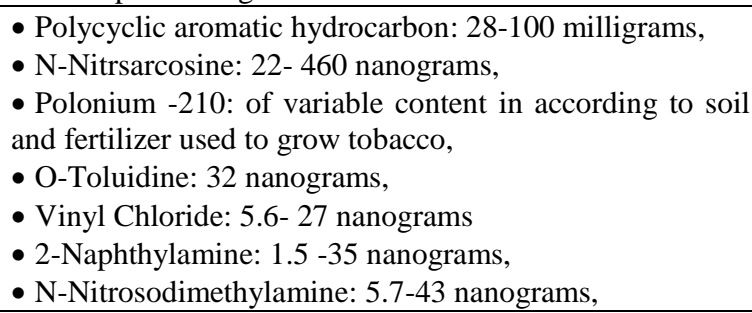

cations that will eventually need further rehabilitation programs in addition to the basic anti-smoking cessation programs.

During the "World's No tobacco Day", on the $31^{\text {st }}$ May, 2018: it was announced that an estimated 8 million persons die every yearworld widely-from smoking effects, and about 600,000 of them were considered as secondary (passive) smokers. During this day, yearly presentations and educational materials are familiarized to the public via 
orientation sessions about hazards of smoking and future advice about active aggressive anti-smoking measures, like considering total ban.

In the US only, there are about $20 \%$ died yearly of the direct smoking effects on the cardiovascular system and many others of about 700,000 persons died from the secondary (passive) smoking effects.

\section{Conclusion and Recommendations}

The smoking per-say is linked to a yearly marked increase of nearly lost lives, illnesses, work absenteeism with a significant negative economical impact worthy losses of US\$ milliards per year, which needs more active focused attention for combating till containment or even an absolute worldwide ban. Smoking is a diseased- habit of all ages of both sexes of different socio-economic class levels, besides that, it is also on the rise, in-spite of, the present efforts, with higher rates in underdeveloped countries due to the lack of health education, especially when associated with more addictive substances dilemma.

To ensure human health safety to all ages and other passive smokers, it is advisable to hold the excessive freedom of harmful smoking desire, by considering totally prohibiting it with stronger compulsory laws of ban, rather than, just a restriction by (not allowed) sign (partial ban) in certain specific area.

Fortunately, usually, when patients stop smoking, it takes few weeks to get rid of smoking withdrawal symptoms like anxiety, cravings, irritability, but, individual patients vary in their response according to strong will, self-motivation and self-control. Many patients when offered the right advise; are well-explained when initially seen, they can get convinced by the harmful effects early and thus can abandon it permanently, without recurrence or medications help. A gain of few kilograms in weight is expected as normal to happen after few weeks' successful abstinence, but might take a period of months-to-years to get rid of the residual systemic harmful circulatory effects of nico- tine, according to packets number per year smoked before, patient's age, whose general condition and individual susceptibility to diseases.

Efforts should be made starting from the early prevention, like getting involved into other healthy activities, instead, as sports or supplying patients with simple substitutes that may contain nicotine in tapered doses as chewing gums, lozenges or search for other equivalents that can have some physiologic dependency effects instead of the harmful psychological-to-pathological dependency patterned effect.

Advisable prophylactic recommendations would be in the form of: 1- WHO suggested raising higher taxes on the smoking industry can help to cut it down. 2- Worldwide smoking details of every patient should be mentioned as part of the routine questionnaire by all hospitals and clinics in all medical specialties for proper screening and early active management i.e. any apparently simple symptom of haemoptysis, a new or worsened chest symptom by a smoker should be taken seriously until proved otherwise, whatever his age is. At least to order a routine chest X-ray and a CT scan. This should be available and offered routinely if possible as a free of charge service by all health supplying facilities. 3- Health education, using different media. 4- Special smoking-cessation programs in specialized individual clinics should be held responsible by all health organizations in an advanced level fashion, as part of an integral clinic included in all hospitals and schools with distinctive- free of chargeillustrative scientific brochures and portable media of handheld data, to be given to all the sick and also the healthy as part of any routine medical management plans. Some of those efforts were deemed highly successful in cutting smoking rates for the last decades by some countries.

5- Worldwide availability of specially licensed and approved ideas and inventions like electronic cigarettes or equivalents, on a cheap or free basis through smoking clinics. 
6- Worldwide availability of Varenecline, the scientific drug name for trade names: (Champix in Europe or, Chantix in the US) is the main US-FDA approved anti-nicotine drug characterized by its ability for specific nicotine receptors in brain with a high success rate in many selected cases. It is a type of aversion therapy to smoking in a few weeks' period, especially if associated with the patient's strong desire of daily tapered off decreasing cigarettes number, to achieve total abstinence. It would be logic to make this widely needed drug more available with cheaper price or else a free supply. 7-Worldwide availability of behavioral-cognitive therapy by more centers, or advanced psychiatric help, especially when mingled with other mixed addictive drugs problem, as some patients usually denies that by confrontation or even asks for the alcoholic anonymous special programs, if needed. Antidotes and psychiatric medications may act or interact differently when combined together. Other possible lung diseases in an addict like TB or diabetes can further immune-compromise unhealthy smoker's lung, which will require further proper added management protocols. 8- Activities, by grand international meetings of smoking at the highest responsible organizations level to agree on certain ban plan details, campaigns, collecting donations and financial aids needed from institutions. 9- Neuroticism and depression were associated with smoking behavior and motives among Egyptians, and thus attention should be given to individual needs in designing and implementing smoking cessation interventions. 10- The majority of the ICU are either smokers or with history of heavy smoking and stopped after sever disease complications. 11- Worldwide collaboration of health authorities over a wide geographic area cross continents after standardized survey mappings of high endemic areas with notification of results to local health authorities and special focused attention are to be made for school children, the grow- ing youth, the addicts, homeless, elderly and highly susceptible patients as cardiac, and those with a history of heavy smoking.

\section{References}

Awadalla, H, Almobarak, AO, Ahmed, MH. 2018: Prevalence of smoking in Sudanese individuals with diabetes and associated complications: Population-based study. Diabetes Metab. Syndr. Apr 24. pii: S1871-4021(18)30148-6.

Ezzeldin, N, El-Lebedy, D, Darwish, A, El Bastawisy, A, Abd Elaziz, SH, et al, 2018: Association of genetic polymorphisms CYP2A6*2 rs 1801272 and CYP2A6*9 rs28399433 with tobacco-induced lung cancer: Case-control study in Egyptian populations. BMC Cancer 18, 1:52-5.

Kenfield, SA, Stampfer, MJ, Rosner, BA, CoIditz, GA, 2008: Smoking and smoking cessation in relation to mortality in women. JAMA 299:2037-47.

Lillard, DR, Önder, Z, 2018: Health information and life-course smoking behavior: evidence from Turkey. Eur. J. Hlth. Econ. 2018 Jun 22. doi: 10.1007/s10198-018-0988-9.

Mohlman, MK, Boulos, DN, El Setouhy, M, Radwan, G, Makambi, K, et al, 2013: A randomized, controlled community-wide intervention to reduce environmental tobacco smoke exposure. Nicotine Tob. Res. 15, 8:1372-81.

Pirie, K, Peto, R, Reeves, GK, Green, J, Beral, V, 2013: Million women study collaborators the $21^{\text {st }}$ Century hazards of smoking and benefits of stopping: a prospective study of one million women in the UK. Lancet 381:133-41.

Verbiest, M, Brakema, E, van der Kleij, R, Sheals, K, Allistone, G, et al, 2017: National guidelines for smoking cessation in primary care: A literature review and evidence analysis. NPJ Prim. Care Respir. Med. 27:2-12.

WHO, 2015: Worker's Health: Global Plan of Action. Available: http://www.who.int/ occupational_health/publications/global_plan/en/. We are:

HAZEM H. M. KHALIL, Hazemkhalil952@gmail.com

MANAL ABDEL-RAHMAN A. MORSY, dr.manal201@gmail.com

AYMAN T. A. MORSY, aymanmorsy72@yahoo.com SOHA MAMDOUH I. NASSAR, sohamamdouh_nassar@yahoo.com 